\title{
An Attempt to Calculate Energy Eigenvalues in Quantum Systems of Large Sizes
}

\author{
T. MUNEHISA and Y.MUNEHISA \\ Faculty of Engineering, Yamanashi University \\ Takeda, Kofu, Yamanashi 400-8511, Japan
}

\begin{abstract}
We report an attempt to calculate energy eigenvalues of large quantum systems by the diagonalization of an effectively truncated Hamiltonian matrix. For this purpose we employ a specific way to systematically make a set of orthogonal states from a trial wavefunction and the Hamiltonian. In comparison with the Lanczos method, which is quite powerful if the size of the system is within the memory capacity of computers, our method requires much less memory resources at the cost of the extreme accuracy.

In this paper we demonstrate that our method works well in the systems of onedimensional frustrated spins up to 48 sites, of bosons on a chain up to 32 sites and of fermions on a ladder up to 28 sites. We will see this method enables us to study eigenvalues of these quantum systems within reasonable accuracy.
\end{abstract}

KEYWORDS: Quantum system, Diagonalization, Large size 


\section{Introduction}

So far a lot of methods have been developed to calculate eigenvalues of large size quantum systems. They can be brought under two chief categories, the exact diagonalization methods [1] and the Monte Carlo approaches [2]. In the former category, where the Lanczos method proves itself to be quite useful, one can expect strictly accurate results as long as the size of a system is small enough. The reason why the size of a system is limited in the Lanczos method is that it consumes enormous memory resources to keep every component of the state. Within this category the density renormalization group method [3] seems most prosperous to study systems of large sizes, but its effectivity strongly depends on both the system's properties and its dimensionality. By means of the Monte Carlo methods, on the other hand, it is possible to study quantum systems with many sites. Instead it becomes necessary to deal with the statistical errors and in some cases the so-called negative sign problem would prevent us to obtain meaningful results. Another disadvantage of the methods is that the dynamical quantities are out of their reach.

In this paper we propose a new method to obtain the eigenvalues in quantum systems of large sizes, which is to be classified into the exact diagonalization approaches. A basic idea is that we calculate expectation values

$$
\left\langle\Psi\left|\hat{O}_{i} \hat{H} \hat{O}_{j}\right| \Psi\right\rangle
$$

with a choice of a trial wavefunction $|\Psi\rangle$ and a set of trial operators $\left\{\hat{O}_{i}\right\}$ aimed to demand much less memory than that required in the Lanczos method, so that the effective Hamiltonian matrix is diagonalized within reasonable computer resources. A good property of our method is that it is applicable to various types of systems.

In the next section we give a brief description of the method, giving general guidelines to find out the trial wavefunction and the trial operators in the systematic way. Concrete expressions for them will be given in the following sections together with the results for the spin, the boson and the fermion systems. The final section is devoted to summary and discussion. 


\section{Method}

In this section we outline our method to calculate the energy eigenvalues and their eigenstates, comparing it with the Lanczos method if necessary.

Let us consider to calculate energy eigenvalues using a finite number of elements of the system's Hamiltonian $\hat{H}$,

$$
H_{i j}=\left\langle\psi_{i}|\hat{H}| \psi_{j}\right\rangle
$$

where the states $\left\{\psi_{1}, \psi_{2}, \cdots, \psi_{n}\right\}$ form a orthonormal set

$$
\left\langle\psi_{i} \mid \psi_{j}\right\rangle=\delta_{i j}
$$

A good choice of the set is essential to obtain accurate eigenvalues using the presently available computer resources.

In the Lanczos method, one begins with an orthogonal set made from an initial state $|\Psi\rangle$,

$$
\left\{|\Psi\rangle, \hat{H}|\Psi\rangle, \hat{H}^{2}|\Psi\rangle, \cdots, \hat{H}^{n-1}|\Psi\rangle\right\}
$$

the value of $n$ being, empirically, of order 100. Then the matrix elements are given by the expectation values $\left\langle\Psi\left|\hat{H}^{k}\right| \Psi\right\rangle$ where $k$ runs from 0 to $2 n-1$. How the CPU time and the memory resources needed to calculate these expectation values increase with the system size $N$ ? To find an answer to this question we assume, for concreteness, that the number of components of each state vector grows as $p^{N}$ with some positive integer $p$ and that the Hamiltonian $\hat{H}$ is the sum of the partial Hamiltonians $\hat{h}_{i}(i=1, \cdots, N)$,

$$
\hat{H}=\sum_{i=1}^{N} \hat{h}_{i},
$$

where $\hat{h}_{i}$ denotes interactions between the site $i$ and the site $i+1$. The CPU time to obtain the expectation values $\left\langle\Psi\left|H^{k}\right| \Psi\right\rangle$ would be of order $N k$ provided that the time to calculate $\hat{h}_{i}|\phi\rangle$ is in the same order for any intermediate state $|\phi\rangle$. It should be noted that the CPU time increases not exponentially but linearly as $N$ increases. The number of the components, on the contrary, will rapidly increase as $(p N)^{k / 2}$ if each $\hat{h}_{i}$ produces a new state. Since one needs to keep every component of 
the states in the evaluation, the exhaustion of the memory resources would prevent one to enlarge the system size $N$.

Now let us introduce our method. We would like to emphasize that this method needs much less memory resources compared with the Lanczos method and moderate, executable CPU time to obtain results on the eigenvalues and the eigenstates within acceptable numerical errors. Instead of the set (1) used in the Lanczos method we employ

$$
\left\{\hat{O}_{0}|\Psi\rangle \equiv|\Psi\rangle, \hat{O}_{1}|\Psi\rangle, \hat{O}_{2}|\Psi\rangle \cdots, \hat{O}_{n-1}|\Psi\rangle\right\}
$$

where these statevectors should be linearly independent with each others and every operator $\hat{O}_{i}$ should have the same symmetry as the Hamiltonian $\hat{H}$ has - the translational invariance, the conservation of the total spin, the conservation of the particle number and so on-so that any state generated from $\hat{O}_{i}$ keeps the related quantum numbers. These requests are, however, too general to find out definite expressions of $\hat{O}_{i}$. Being strongly motivated by the success of the Lanczos method we additively demand that the expectation values $\left\langle\Psi\left|\hat{H}^{k}\right| \Psi\right\rangle$ can be, in principle, accurately evaluated with the set $\left\{|\Psi\rangle, \hat{O}_{1}|\Psi\rangle, \hat{O}_{2}|\Psi\rangle \cdots, \hat{O}_{n-1}|\Psi\rangle\right\}$. Thus we find a hint for a systematic choice of $\left\{\hat{O}_{i}\right\}$ in the expression obtained using (2),

$$
\left\langle\Psi\left|\hat{H}^{k}\right| \Psi\right\rangle=\sum_{i_{1}=1}^{N} \sum_{i_{2}=1}^{N} \cdots \sum_{i_{k}=1}^{N}\left\langle\Psi\left|\hat{h}_{i_{1}} \hat{h}_{i_{2}} \cdots \hat{h}_{i_{k}}\right| \Psi\right\rangle .
$$

Since the number of components one needs to calculate each $\left\langle\Psi\left|\hat{h}_{i_{1}} \hat{h}_{i_{2}} \cdots \hat{h}_{i_{k}}\right| \Psi\right\rangle$ is of the order of $p^{k / 2}$, it seems favorable for our purpose to select the operators $\left\{\hat{O}_{i}\right\}$ from $\left\{\hat{h}_{i_{1}} \hat{h}_{i_{2}} \cdots \hat{h}_{i_{k}}\right\}$. Although we can deal with, of course, only a small portion of the whole $\left\{\hat{h}_{i_{1}} \hat{h}_{i_{2}} \cdots \hat{h}_{i_{k}}\right\}$ for systems of large sizes because the number of terms in the sum increases as $N^{k}$, we will see it is often the case that such $\left\{\hat{O}_{i}\right\}$ is enough to obtain the satisfying results.

As for the initial state $|\Psi\rangle$, the symmetry of the state we want to study - the lowest energy state for example - is useful to determine it. It is desirable to choose a $|\Psi\rangle$ possessing the same quantum numbers as the state to be studied. The restructuring method [4] for re-arrangements of states is, in addition, quite helpful to get the $|\Psi\rangle$ which effectively reduces the CPU time in the calculation. 
Once the operators $\left\{\hat{O}_{i}\right\}$ and the initial state $|\Psi\rangle$ are determined for the system under consideration, the rest of the calculation is conventional. We calculate the Hamiltonian matrix $H_{O i j}(i, j=1, \cdots, n)$,

$$
H_{O i j} \equiv\left\langle\Psi\left|\hat{O}_{i-1} \hat{H} \hat{O}_{j-1}\right| \Psi\right\rangle
$$

Then, employing the Gram-Schmidt method to create an orthonormal set $\left\{\left|\psi_{1}\right\rangle, \cdots,\left|\psi_{n}\right\rangle\right\}$, we obtain the coefficients $t_{k i}$,

$$
\left|\psi_{k}\right\rangle=\sum_{i=1}^{k} \hat{O}_{i-1}|\Psi\rangle t_{k i}
$$

which are utilized to calculate the effective Hamiltonian matrix $\bar{H}_{O i j}$,

$$
\bar{H}_{O i j}=\sum_{i^{\prime}=1}^{n} \sum_{j^{\prime}=1}^{n} H_{O i^{\prime} j^{\prime}} t_{i i^{\prime}} t_{j j^{\prime}},
$$

where $t_{k i} \equiv 0$ for $i>k$. The resultant $\bar{H}_{O i j}$ can be easily diagonalized by means of the traditional numerical methods.

\section{Spin system}

In this section we apply our method to a quantum spin system. We employ the one-dimensional frustrated system which has both the nearest-neighbor and the next-nearest-neighbor interactions, concentrating our attention to its ground state. The reasons why we adopt this system here are that its ground state is non-trivial and that the exact energy eigenvalues obtained by other methods are available for the system of small sizes.

The Hamiltonian we study is

$$
\hat{H}=\sum_{i=1}^{N^{\prime}} \vec{\sigma}_{a, i} \vec{\sigma}_{a, i+1}+\sum_{i=1}^{N^{\prime}} \vec{\sigma}_{b, i} \vec{\sigma}_{b, i+1}+\sum_{i=1}^{N^{\prime}} \vec{\sigma}_{a, i} \vec{\sigma}_{b, i}+\sum_{i=1}^{N^{\prime}} \vec{\sigma}_{b, i} \vec{\sigma}_{a, i+1},
$$

where the suffix $a(b)$ denotes the odd (even) sites on a chain with $N \equiv 2 N^{\prime}$ spins and the periodic boundary condition $\vec{\sigma}_{a(b), N^{\prime}+1} \equiv \vec{\sigma}_{a(b), 1}$ is imposed. 
First let us discuss on the initial state $|\Psi\rangle$. In conventional calculations each state of the system is represented by $\left|s_{a, 1}, s_{b, 1}, s_{a, 2}, s_{b, 2}, \cdots, s_{a, N^{\prime}}, s_{b, N^{\prime}}\right\rangle$ with each $s_{a(b), i}= \pm$, which indicates that the $z$ component of the spin is $\pm 1 / 2$. In the restructuring method [4] we rearrange states $s_{a, i}$ and $s_{b, i}$ into a singlet state $\left|\ominus_{i}\right\rangle$ and three triplet states $\left|1_{i}\right\rangle,\left|\oplus_{i}\right\rangle$ and $\left|-1_{i}\right\rangle$,

$$
\begin{aligned}
\left|\ominus_{i}\right\rangle & =\frac{1}{\sqrt{2}}\left(\left|+_{a, i},-{ }_{b, i}\right\rangle-\left|-_{a, i},+_{b, i}\right\rangle\right), \\
\left|1_{i}\right\rangle & =\left|+_{a, i},+_{b, i}\right\rangle, \\
\left|\oplus_{i}\right\rangle & =\frac{1}{\sqrt{2}}\left(\left|++_{a, i},--_{b, i}\right\rangle+\left|--_{a, i},+_{b, i}\right\rangle\right), \\
\left|-1_{i}\right\rangle & =\left|--_{a, i},-_{b, i}\right\rangle,
\end{aligned}
$$

which we inclusively denote by $\left|S_{i}\right\rangle$. The complete set for the states of the system is now given by $\left\{\left|S_{1}, S_{2}, \cdots, S_{N^{\prime}}\right\rangle\right\}$. Since the spin-singlet state in this set is expressed by only one component we employ this as the initial state,

$$
|\Psi\rangle=\left|\ominus_{1}, \ominus_{2}, \cdots, \ominus_{N^{\prime}}\right\rangle
$$

so that we can largely reduce the CPU time needed in the calculation. It should be minded that the contamination of the states with momentum $\pi$ is an undesirable feature of this choice, because the ground state should have the zero momentum only. In spite of this fact, however, we will see we can obtain satisfying results with this $|\Psi\rangle$.

Next we construct the operators $\left\{\hat{O}_{i}\right\}$ from $\hat{H}^{k}$. Taking the restructuring method into account, we resolve the Hamiltonian $\hat{H}$ into its components $\vec{\sigma}_{a, i} \vec{\sigma}_{b, i}$ and $\hat{h}_{i} \equiv$ $\vec{\sigma}_{a, i} \vec{\sigma}_{a, i+1}+\vec{\sigma}_{b, i} \vec{\sigma}_{b, i+1}+\vec{\sigma}_{b, i} \vec{\sigma}_{a, i+1}$,

$$
\hat{H}=\sum_{i=1}^{N^{\prime}} \vec{\sigma}_{a, i} \vec{\sigma}_{b, i}+\sum_{i=1}^{N^{\prime}} \hat{h}_{i} .
$$

Then in the expansion of $\hat{H}^{k}$ we direct our attention to the terms which are products of several $\hat{h}_{i}$ 's with the suffix $i$ being in the whole range $1 \leq i \leq N^{\prime}$. We combine those terms to express the candidates for $\left\{\hat{O}_{i}\right\}$, which are described by

$$
\hat{O}\left(k_{1}, k_{2}, \cdots, k_{L}\right) \equiv \sum_{i=1}^{N^{\prime}} \hat{h}_{i+k_{1}} \hat{h}_{i+k_{2}} \cdots \hat{h}_{i+k_{L}}
$$


with some positive integer $L$, which we will call the order of the operator hereafter, and some positive integers $k_{2}, k_{3}, \cdots, k_{L}$. Here we keep $k_{1} \equiv 0$.

Now we show the numerical results. First let us report that this method presents the exact eigenvalues for the system of small sizes up to $N=12$. For $N=8$ the set of the states

$$
\left\{|\Psi\rangle, \hat{O}_{1}|\Psi\rangle, \cdots, \hat{O}_{5}|\Psi\rangle\right\}
$$

with

$$
|\Psi\rangle=\left|\ominus_{1}, \ominus_{2}, \ominus_{3}, \ominus_{4}\right\rangle,
$$

and

$$
\hat{O}_{1}=\hat{O}(0), \quad \hat{O}_{2}=\hat{O}(0,1), \quad \hat{O}_{3}=\hat{O}(0,2), \quad \hat{O}_{4}=\hat{O}(0,1,0), \quad \hat{O}_{5}=\hat{O}(0,1,2)
$$

gives us the exact eigenvalues'.

When $N=12$ the exact eigenvalue is obtainable with 28 states selected in the similar way. Table 1 presents the list of the operators $\hat{O}_{i}$ in this case. In order to see, without using other methods, whether the calculated eigenvalue of $\hat{H}$ is exact, we need to calculate the corresponding eigenvalue of $\hat{H}^{2}$, too. If the latter value agrees with the square of the former, the result is exact. Table 2 shows that the differences between the two for the $N=12$ chain are negligible.

In the cases of larger sizes we have to do with approximate eigenvalues obtained from a limited number of the operators. To make a systematic selection of $\hat{O}_{i}$ from its candidates, we gradually increase the value of the order of the operator $L$ in Eq.(9). Suppose that we have already picked up the operators up to, say, $L \leq L_{0}$. The next step of the procedure is to list up $\hat{O}\left(k_{1}, k_{2}, \cdots, k_{L_{0}+1}\right)$ 's with possible values of $\left\{k_{2}, k_{3}, \cdots, k_{L_{0}+1}\right\}\left(k_{1} \equiv 0\right)$ and add each of them into the set of $\left\{\hat{O}_{i}\right\}$ if it creates a new state, namely a state which is linearly independent of all the previously chosen $\hat{O}_{i}|\Psi\rangle$ 's, when operated to the initial state $|\Psi\rangle$. The operators in Table 1 also have been generated by this way. Note that the whole procedure is carried out by a computer program so that any work by hand is not necessary here.

\footnotetext{
${ }^{1}$ Note that the number of the states necessary for the set also strongly depends on the system's property. For the quantum spin system on a ladder where there are no cross terms $\vec{\sigma}_{b, i} \vec{\sigma}_{a, i+1}$, for instance, three states are enough to obtain the exact eigenvalue.
} 
In Table 3 we show the approximate eigenvalues of the ground state energy in the case of $N=48$ for $L \leq 5$, together with the exact one obtained by the Lanczos method [1]. We see the approximation is improved as we pick up more operators bearing the cost of the longer CPU time, which is mainly originated from the increasing number of the states. In other words, in order to investigate systems of larger sizes within the available CPU time we have to adopt less precise results. So the maximum size of the system is determined by arranging a compromise between these factors. The size $N=48$ is the largest one we study in this paper.

\section{Boson system}

Now let us turn our attention to boson systems with the invariant boson number $N_{b}$. We consider the one-dimensional system with $N$ sites, whose Hamiltonian is given by

$$
\hat{H}=-t \sum_{i=1}^{N}\left(\hat{a}_{i+1}^{\dagger} \hat{a}_{i}+\hat{a}_{i}^{\dagger} \hat{a}_{i+1}\right)+\lambda \sum_{i=1}^{N}\left(\hat{a}_{i}^{\dagger} \hat{a}_{i}\right)^{2},
$$

$\hat{a}_{i}\left(\hat{a}_{i}^{\dagger}\right)$ being an annihilation (a creation) operator on the site $i$, with the periodic boundary condition $\hat{a}_{N+1}^{(\dagger)} \equiv \hat{a}_{1}^{(\dagger)}$. In the numerical work we take $t \equiv 1$, while $\lambda=4$ unless stated otherwise.

Limiting ourselves to the case $N_{b}=N$ we employ a translational invariant state $|1,1, \cdots, 1\rangle$, where each site of the chain has one boson on it, as the initial state $|\Psi\rangle$. Note that we expect this is a good choice for large values of the coupling $\lambda$ since the state is the exact ground state in the strong coupling limit $\lambda \rightarrow \infty$.

As for the operators $\left\{\hat{O}_{i}\right\}$, we follow the same procedure as previously stated in Section 3 to select them from the candidates

$$
\hat{O}\left(k_{1}, k_{2}, \cdots, k_{L}\right) \equiv \sum_{i=1}^{N} \hat{g}_{i+k_{1}} \hat{g}_{i+k_{2}} \cdots \hat{g}_{i+k_{L}}
$$

where we use the notation $\hat{g}_{i} \equiv \hat{a}_{i}^{\dagger} \hat{a}_{i+1}+\hat{a}_{i+1}^{\dagger} \hat{a}_{i}$. The reason why we do not include the diagonal terms of the Hamiltonian in $\hat{g}_{i}$ is that more of the inner products 
$\left\langle\Psi\left|\hat{O}_{i} \hat{O}_{j}\right| \Psi\right\rangle$ will become zero without the diagonal terms.

Figs. 1 and 2 present the results on the system's ground state energy. In Fig. 1 we plot the errors in our calculations for the $N=8$ system, which is small enough to obtain the exact value using the Lanczos method, as a function of the coupling $\lambda$. The results indicate that our method works well in a wide range of the parameter space. Especially in the strong coupling region we see the agreement is excellent, which confirms our expectation that the initial state we employed is good there. Fig. 2 shows our results on chains up to $N=32$, together with the exact values for $N \leq 10$. The discrepancy between the "exact" value, which we guess from the exact values obtained on the short chains, and our result is about $0.5 \%$ (1\%) for $N=16$ (32), respectively. These errors could be lessened by including the higher-order operators with $L \geq 5$.

\section{Fermion system}

This section is devoted to the study of two fermion systems, the standard Hubbard model and the extended Hubbard model which has been extensively studied by the authors using a quantum Monte Carlo method [5]. Here we limit ourselves only to the ground state of the half-filled system. We will show our method nicely works for these systems, too.

The Hamiltonian of the extended Hubbard model we study is

$$
\begin{array}{r}
\hat{H}=-t_{c} \sum_{\sigma} \sum_{l} \sum_{i=1}^{N_{r}}\left[c_{i, l, \sigma}^{\dagger} c_{i+1, l, \sigma}+h . c .\right]-t_{r} \sum_{\sigma} \sum_{i=1}^{N_{r}}\left[c_{i, a, \sigma}^{\dagger} c_{i, b, \sigma}+h . c .\right] \\
+V_{c} \sum_{\sigma, \sigma^{\prime}} \sum_{l} \sum_{i=1}^{N_{r}-1} n_{i, l, \sigma} n_{i+1, l, \sigma^{\prime}}+V_{r} \sum_{\sigma, \sigma^{\prime}} \sum_{i=1}^{N_{r}} n_{i, a, \sigma} n_{i, b, \sigma^{\prime}}+U \sum_{l} \sum_{i=1}^{N_{r}} n_{i, l, \uparrow} n_{i, l, \downarrow},
\end{array}
$$

where $c_{i, l, \sigma}^{(\dagger)}$ denotes the annihilation (creation) operator for an electron, whose spin being $\sigma(\uparrow$ or $\downarrow)$, located on the $i$-th rung along the leg $l$ ( $a$ or $b$ ) of a ladder which has $N_{r}$ rungs in total and $n$ is the number operator $n_{i, l, \sigma} \equiv c_{i, l, \sigma}^{\dagger} c_{i, l, \sigma}$. We assume the periodic boundary conditions $c_{N_{r}+1, l, \sigma}^{(\dagger)} \equiv c_{1, l, \sigma}^{(\dagger)}$. The standard Hubbard model is 
described by setting parameters $V_{r}=V_{c}=0$.

In the calculation we employ the restructuring method. We use a complete set $\{|\alpha\rangle\}$, where

$$
|\alpha\rangle=\left|S_{1}, S_{2}, \cdots, S_{N_{r}}\right\rangle
$$

with sixteen states in Table 4 to denote the state $S_{i}$ on the $i$-th rung]. It should be noted that the states in the table are eigenstates for the interaction terms between two sites on the $i$-th rung, namely eigenstates for

$$
-t_{r} \sum_{\sigma}\left[c_{i, a, \sigma}^{\dagger} c_{i, b, \sigma}+\text { h.c. }\right]+V_{r} \sum_{\sigma, \sigma^{\prime}} n_{i, a, \sigma} n_{i, b, \sigma^{\prime}}+U \sum_{l} n_{i, l, \uparrow} n_{i, l, \downarrow} .
$$

The operator $\hat{O}_{i}$ is the product of

$$
\hat{f}_{i} \equiv-t_{c} \sum_{\sigma} \sum_{l}\left[c_{i, l, \sigma}^{\dagger} c_{i+1, l, \sigma}+\text { h.c. }\right]+V_{c} \sum_{\sigma, \sigma^{\prime}} \sum_{l} n_{i, l, \sigma} n_{i+1, l, \sigma^{\prime}}
$$

which comes from the interaction terms along the chains, selected from the candidates

$$
\hat{O}\left(k_{1}, k_{2}, \cdots, k_{L}\right) \equiv \sum_{i=1}^{N} \hat{f}_{i+k_{1}} \hat{f}_{i+k_{2}} \cdots \hat{f}_{i+k_{L}} .
$$

First let us report the results for the standard Hubbard model. In this case we choose as an initial state $|\Psi\rangle$ a translational invariant state whose all $S_{i}$ are No. 9 in Table 4. We plot the results on the ground state energy per site in Fig. 3 as a function of the maximum value of $L, L_{\max }$, for several values of $N\left(=2 N_{r}\right)$ up to 28. Here the values of the active parameters in (12) are set to be $t_{c}=1, t_{r}=2$ and $U=4$, respectively. We see that the values in Fig. 3 nicely converge into a value around -1.269 . We would like to emphasize that this value is compatible with our newly calculated quantum Monte Carlo result for the same Hamiltonian with the different boundary conditions. Although we have to accept the poor statistical

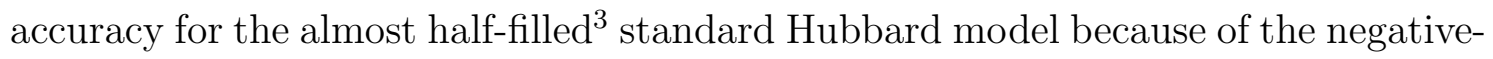
sign problem, we obtain the value $-1.26 \pm 0.02$ at the inverse temperature $\beta=10$ on an $N=32$ ladder with the open boundary conditions.

\footnotetext{
2 Parameters $u_{1}$ and $u_{2}$ in Table 4 are functions of $t_{r}, V_{r}$ and $U$ given by $u_{1} \equiv \frac{1}{2} \sqrt{1+\frac{U-V_{r}}{\sqrt{\left(U-V_{r}\right)^{2}+16 t_{r}^{2}}}}$ and $u_{2} \equiv \frac{1}{2} \sqrt{1-\frac{U-V_{r}}{\sqrt{\left(U-V_{r}\right)^{2}+16 t_{r}^{2}}}}$

${ }^{3}$ In the Monte Carlo study the number of the fermions can slightly fluctuate according to the given value of the chemical potential.
} 
Next we show the results on the extended Hubbard model with parameters $t_{c}=1$, $t_{r}=2, V_{c}=2, V_{r}=-4$ and $U=4$ in (12). In our previous quantum Monte Carlo study [5] it turned out that the negative-sign problem is not serious for these values of the parameters in a wide range of the chemical potential and that the system indicates a signal to the phase separation. Since we have learned from this study that the ground state near the half-filled system is abundant in both the hole pairs on the rungs and the doubly occupied sites, we choose the initial state in which the state on each rung $i$ is No. 1 (No. 16) in Table 4 for even (odd) $i$. In Fig. 4 we plot the results for the ground state energy per site on the even $N_{r}$ ladders obtained with such initial states. They also show nice convergence and are close to the exact value -2.202 on the $N=8$ ladder with the periodic boundary conditions. The quantum Monte Carlo result for the mostly half-filled case of the model is $-2.234 \pm 0.007$ (statistical error only) with $\beta=5$ and $N=16$ under the open boundary conditions.

\section{Summary and Discussion}

In this paper we showed an attempt to calculate eigenvalues of the Hamiltonian in various quantum systems of large sizes. In the calculation we start from a trial wavefunction, which we should choose carefully taking account of the symmetry of the state to be studied. Then we make a set of states by repeatedly applying some

operators $\left\{\hat{O}_{i}\right\}$ to the trial wavefunction. Our basic idea is to determine each $\hat{O}_{i}$ systematically using several terms in the Hamiltonian of the system under consideration. Then we select a limited number of states to construct an effective matrix of the Hamiltonian, whose eigenvalues are easily obtained by the conventional diagonalization methods. In our calculation restrictions from the memory resources of computers are relaxed, while the available CPU time sets bounds to the obtainable accuracy and the reachable maximum system size. As concrete examples we demonstrate the results for low-dimensional spin, boson and fermion systems. We 
find the method works satisfactorily for all cases we studied.

In order to pursue further precise numerical results or to apply our method to larger systems including higher-dimensional ones, however, we need to achieve some more improvements. One of them concerns with the size of the effective Hamiltonian matrix. Since we select states from $H^{k}|\Psi\rangle$, the number of the candidate states rapidly increases as either the power $k$ or the size of the system becomes large. For the purposes stated above, therefore, we would be obliged to construct the larger effective Hamiltonian matrices for which serious restrictions again would come from the available memory resources. So we should develop an effective method to treat large size matrices. Another problem is that, because of the rapidly increasing CPU time, it is difficult to increase the order of the operators beyond the values we have used here. A possible breakthrough for it would be to develop techniques to predict which of the states are linearly dependent.

\section{Acknowledgement}

We would like to thank Prof. Nishimori for his programs on the diagonalization. 


\section{References}

[1] E. Dagotto: Rev. of Mod. Phys. Vol. 66 (1994) 763.

E. Dagotto and A. Moreo: Phys. Rev. D21 (1985) 865.

E. S. Heeb and T. M. Rice: Z. Phys. B90 (1993) 73.

E. Gaglino, E. Dagotto, A. Moreo and F. Alcaraz: Phys. Rev. B34 (1986) 1677.

H. Nishimori and H. Nakanishi: J. Phys. Soc. Jpn. 57 (1990) 4454.

[2] N. Hatano and M. Suzuki: Quantum Monte Carlo Methods in Condensed Matter

Physics, ed. M. Suzuki (World Scientific, Singapore, 1993) p.13.

[3] S. R. White: Phys. Rev. Lett. 69 (1992) 2863;

Phys. Rev. B48 (1993) 10345.

[4] T. Munehisa and Y. Munehisa: Phys. Rev. B49 (1994) 3347;

Prog. Theor. Phys. 92 (1994) 309;

Prog. Theor. Phys. 93 (1995) 251;

Prog. Theor. Phys. 96 (1996) 59.

[5] T. Munehisa and Y. Munehisa: J. Phys. Soc. Jpn. 66 (1997) 3876. 


\begin{tabular}{|c|c|ccccc||c|c|ccccc|}
\hline No. & Order & $k_{1}$ & $k_{2}$ & $k_{3}$ & $k_{4}$ & $k_{5}$ & No. & Order & $k_{1}$ & $k_{2}$ & $k_{3}$ & $k_{4}$ & $k_{5}$ \\
\hline \hline 1 & 0 & - & - & - & - & - & 15 & 4 & 0 & 1 & 0 & 5 & - \\
\hline 2 & 1 & 0 & - & - & - & - & 16 & 4 & 0 & 1 & 2 & 1 & - \\
\hline 3 & 2 & 0 & 1 & - & - & - & 17 & 4 & 0 & 1 & 2 & 3 & - \\
\hline 4 & 2 & 0 & 2 & - & - & - & 18 & 4 & 0 & 1 & 2 & 4 & - \\
\hline 5 & 2 & 0 & 3 & - & - & - & 19 & 4 & 0 & 1 & 3 & 2 & - \\
\hline 6 & 3 & 0 & 1 & 0 & - & - & 20 & 4 & 0 & 1 & 3 & 4 & - \\
\hline 7 & 3 & 0 & 1 & 2 & - & - & 21 & 4 & 0 & 2 & 1 & 4 & - \\
\hline 8 & 3 & 0 & 1 & 3 & - & - & 22 & 5 & 0 & 1 & 0 & 2 & 3 \\
\hline 9 & 3 & 0 & 1 & 4 & - & - & 23 & 5 & 0 & 1 & 0 & 2 & 4 \\
\hline 10 & 3 & 0 & 2 & 1 & - & - & 24 & 5 & 0 & 1 & 0 & 3 & 2 \\
\hline 11 & 3 & 0 & 2 & 4 & - & - & 25 & 5 & 0 & 1 & 0 & 3 & 4 \\
\hline 12 & 4 & 0 & 1 & 0 & 2 & - & 26 & 5 & 0 & 1 & 2 & 1 & 4 \\
\hline 13 & 4 & 0 & 1 & 0 & 3 & - & 27 & 5 & 0 & 1 & 2 & 3 & 4 \\
\hline 14 & 4 & 0 & 1 & 0 & 4 & - & 28 & 5 & 0 & 2 & 1 & 4 & 3 \\
\hline
\end{tabular}

Table 1: Operators $\hat{O}\left(k_{1}, k_{2}, \cdots, k_{L}\right)$ 's needed for the spin system on the $N=12$ chain. See (9) for the definition of the operator. 


\begin{tabular}{|c|c|c|c||c|c|c|c|}
\hline No. & $\hat{H}$ & $\hat{H}^{2}$ & Diff. & No. & $\hat{H}$ & $\hat{H}^{2}$ & Diff. \\
\hline \hline 1 & -23.585 & 556.24 & $0.37064 \mathrm{E}-07$ & 15 & -7.0086 & 49.121 & $0.82701 \mathrm{E}-08$ \\
\hline 2 & -22.901 & 524.44 & $0.37073 \mathrm{E}-07$ & 16 & -6.9468 & 48.258 & $0.25527 \mathrm{E}-07$ \\
\hline 3 & -18.409 & 338.90 & $0.46285 \mathrm{E}-07$ & 17 & -4.2793 & 18.313 & $0.15361 \mathrm{E}-07$ \\
\hline 4 & -17.271 & 298.29 & $0.27047 \mathrm{E}-07$ & 18 & -4.0000 & 16.000 & $0.10467 \mathrm{E}-07$ \\
\hline 5 & -15.904 & 252.93 & $0.82821 \mathrm{E}-09$ & 19 & -3.4452 & 11.869 & $0.41185 \mathrm{E}-08$ \\
\hline 6 & -13.904 & 193.31 & $0.29984 \mathrm{E}-07$ & 20 & -1.5279 & 2.3344 & $0.98762 \mathrm{E}-10$ \\
\hline 7 & -13.411 & 179.85 & $0.44405 \mathrm{E}-07$ & 21 & -1.2330 & 1.5204 & $0.10958 \mathrm{E}-08$ \\
\hline 8 & -12.000 & 144.00 & $0.26574 \mathrm{E}-07$ & 22 & -0.69308 & 0.48036 & $0.13380 \mathrm{E}-08$ \\
\hline 9 & -11.446 & 131.00 & $0.42669 \mathrm{E}-07$ & 23 & 0.47600 & 0.22658 & $0.14751 \mathrm{E}-08$ \\
\hline 10 & -11.266 & 126.92 & $0.33501 \mathrm{E}-08$ & 24 & 3.3595 & 11.286 & $0.52512 \mathrm{E}-08$ \\
\hline 11 & -10.472 & 109.67 & $0.22215 \mathrm{E}-07$ & 25 & 4.8753 & 23.768 & $0.89002 \mathrm{E}-08$ \\
\hline 12 & -10.065 & 101.29 & $0.31402 \mathrm{E}-09$ & 26 & 5.9206 & 35.054 & $0.53468 \mathrm{E}-09$ \\
\hline 13 & -8.0485 & 64.778 & $0.26372 \mathrm{E}-07$ & 27 & 10.167 & 103.37 & $0.16889 \mathrm{E}-07$ \\
\hline 14 & -7.6605 & 58.684 & $0.26150 \mathrm{E}-07$ & 28 & 16.676 & 278.10 & $0.32337 \mathrm{E}-07$ \\
\hline
\end{tabular}

Table 2: Eigenvalues obtained in our calculations for the $N=12$ chain. Here the operators of the order $L \leq 5$ are used. The column of $\hat{H}$ shows the eigenvalues of the truncated Hamiltonian matrix, while those from the Hamiltonian squared are shown in the column of $\hat{H}^{2}$. The third value is the difference between the second value and the square of the first one. 


\begin{tabular}{|c|c|c|c|c|}
\hline Size & Maximum order & Num. of state & $\mathrm{E}$ & $\mathrm{E}$ (exact) \\
\hline \hline 16 & 4 & 37 & -1.935 & -1.94010 \\
\hline 20 & 4 & 59 & -1.928 & -1.93565 \\
\hline 24 & 4 & 92 & -1.920 & - \\
\hline 28 & 4 & 134 & -1.911 & - \\
\hline 32 & 4 & 191 & -1.902 & - \\
\hline 36 & 4 & 263 & -1.892 & - \\
\hline 40 & 4 & 352 & -1.883 & - \\
\hline 44 & 4 & 460 & -1.874 & - \\
\hline 48 & 4 & 591 & -1.865 & - \\
\hline \hline 12 & 5 & 28 & -1.96538 & -1.96538 \\
\hline 16 & 5 & 78 & -1.9040 & -1.94010 \\
\hline
\end{tabular}

Table 3: Eigenvalues of the ground state for the truncated Hamiltonian matrix up to $N \leq 48$. The second column (Maximum order) indicates the maximum value of $L$. 


\begin{tabular}{|r|c|}
\hline No. & state \\
\hline 1 & $|00\rangle$ \\
\hline 2 & $\frac{1}{\sqrt{2}}\left(c_{i, a, \uparrow}^{\dagger}+c_{i, b, \uparrow}^{\dagger}\right)|00\rangle$ \\
\hline 3 & $\frac{1}{\sqrt{2}}\left(c_{i, a, \uparrow}^{\dagger}-c_{i, b, \uparrow}^{\dagger}\right)|00\rangle$ \\
\hline 4 & $\frac{1}{\sqrt{2}}\left(c_{i, a, \downarrow}^{\dagger}+c_{i, b, \downarrow}^{\dagger}\right)|00\rangle$ \\
\hline 5 & $\frac{1}{\sqrt{2}}\left(c_{i, a, \downarrow}^{\dagger}-c_{i, b, \downarrow}^{\dagger}\right)|00\rangle$ \\
\hline 6 & $c_{i, a, \uparrow}^{\dagger} c_{i, b, \uparrow}|00\rangle$ \\
\hline 7 & $\frac{1}{\sqrt{2}}\left(c_{i, a, \uparrow}^{\dagger} c_{i, b, \downarrow}^{\dagger}+c_{i, a, \downarrow}^{\dagger} c_{i, b, \uparrow}^{\dagger}\right)|00\rangle$ \\
\hline 8 & $\frac{1}{\sqrt{2}}\left(c_{i, a, \uparrow}^{\dagger} c_{i, a, \downarrow}^{\dagger}-c_{i, b, \uparrow}^{\dagger} c_{i, b, \downarrow}^{\dagger}\right)|00\rangle$ \\
\hline 9 & {$\left[u_{1}\left(c_{i, a, \uparrow}^{\dagger} c_{i, b, \downarrow}^{\dagger}-c_{i, a, \downarrow}^{\dagger} c_{i, b, \uparrow}^{\dagger}\right)+u_{2}\left(c_{i, a, \uparrow}^{\dagger} c_{i, a, \downarrow}^{\dagger}+c_{i, b, \uparrow}^{\dagger} c_{i, b, \downarrow}^{\dagger}\right)\right]|00\rangle$} \\
\hline 10 & {$\left[u_{2}\left(c_{i, a, \uparrow}^{\dagger} c_{i, b, \downarrow}^{\dagger}-c_{i, a, \downarrow}^{\dagger} c_{i, b, \uparrow}^{\dagger}\right)-u_{1}\left(c_{i, a, \uparrow}^{\dagger} c_{i, a, \downarrow}^{\dagger}+c_{i, b, \uparrow}^{\dagger} c_{i, b, \downarrow}^{\dagger}\right)\right]|00\rangle$} \\
\hline 11 & $c_{i, a, \downarrow}^{\dagger} c_{i, b, \downarrow}^{\dagger}|00\rangle$ \\
\hline 12 & $\frac{1}{\sqrt{2}}\left(c_{i, a, \uparrow}^{\dagger} c_{i, a, \downarrow}^{\dagger} c_{i, b, \uparrow}^{\dagger}+c_{i, a, \uparrow}^{\dagger} c_{i, b, \uparrow}^{\dagger} c_{i, b, \downarrow}^{\dagger}\right)|00\rangle$ \\
\hline 13 & $\frac{1}{\sqrt{2}}\left(c_{i, a, \uparrow}^{\dagger} \uparrow c_{i, a, \downarrow}^{\dagger} c_{i, b, \uparrow}^{\dagger}-c_{i, a, \uparrow}^{\dagger} c_{i, b, \uparrow}^{\dagger} c_{i, b, \downarrow}^{\dagger}\right)|00\rangle$ \\
\hline 14 & $\frac{1}{\sqrt{2}}\left(c_{i, a, \uparrow}^{\dagger} c_{i, a, \downarrow}^{\dagger} c_{i, b, \downarrow}^{\dagger}+c_{i, a, \downarrow}^{\dagger} c_{i, b, \uparrow}^{\dagger} c_{i, b, \downarrow}^{\dagger}\right)|00\rangle$ \\
\hline 15 & $\frac{1}{\sqrt{2}}\left(c_{i, a, \uparrow}^{\dagger} \uparrow c_{i, a, \downarrow}^{\dagger} c_{i, b, \downarrow}^{\dagger}-c_{i, a, \downarrow}^{\dagger} c_{i, b, \uparrow}^{\dagger} c_{i, b, \downarrow}^{\dagger}\right)|00\rangle$ \\
\hline 16 & $c_{i, a, \uparrow}^{\dagger} c_{i, a, \downarrow}^{\dagger} c_{i, b, \uparrow}^{\dagger} c_{i, b, \downarrow}^{\dagger}|00\rangle$ \\
\hline
\end{tabular}

Table 4: States on two sites of the $i$-th rung used to construct a complete set. $|00\rangle$ represents a state with no electrons on either site of the rung. Parameters $u_{1}$ and $u_{2}$ are functions of $t_{r}, V_{r}$ and $U$ defined in Section 5. 


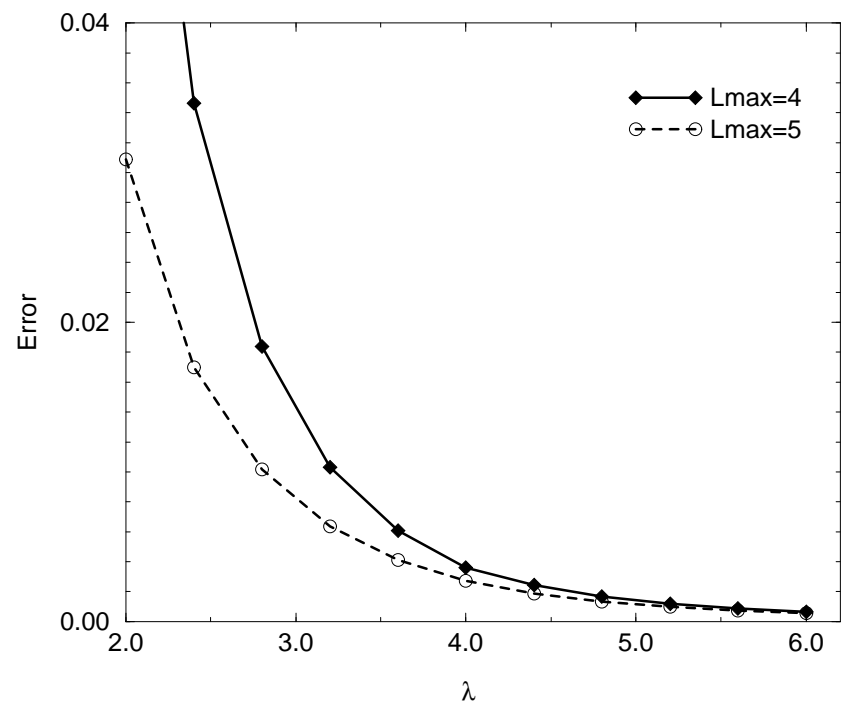

Figure 1: Errors of the calculated eigenvalues of the ground state plotted for several values of the coupling $\lambda$ on the $N=8$ chain, which are defined by the ratio $\left(E_{\text {cal }}-E_{\text {exact }}\right) / E_{\text {exact }}$. The diamonds show the errors for the order $L \leq 4$, while the circles are for the order $L \leq 5$. 


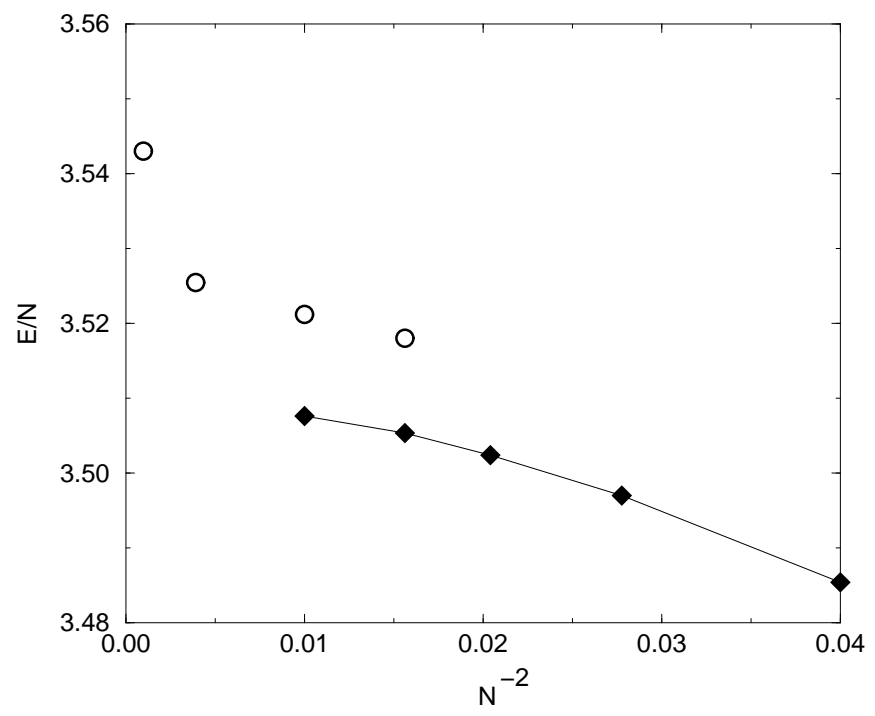

Figure 2: Eigenvalues of the ground state per site (the circles) calculated for $N=8,10,16$ and 32 chains with the coupling $\lambda=4$ and the order $L \leq 4$. The data are plotted versus $N^{-2}$. The number of the states is 304 (1844) for $N=16$ (32), respectively. Exact values for the systems up to $N \leq 10$ are also shown by the diamonds.

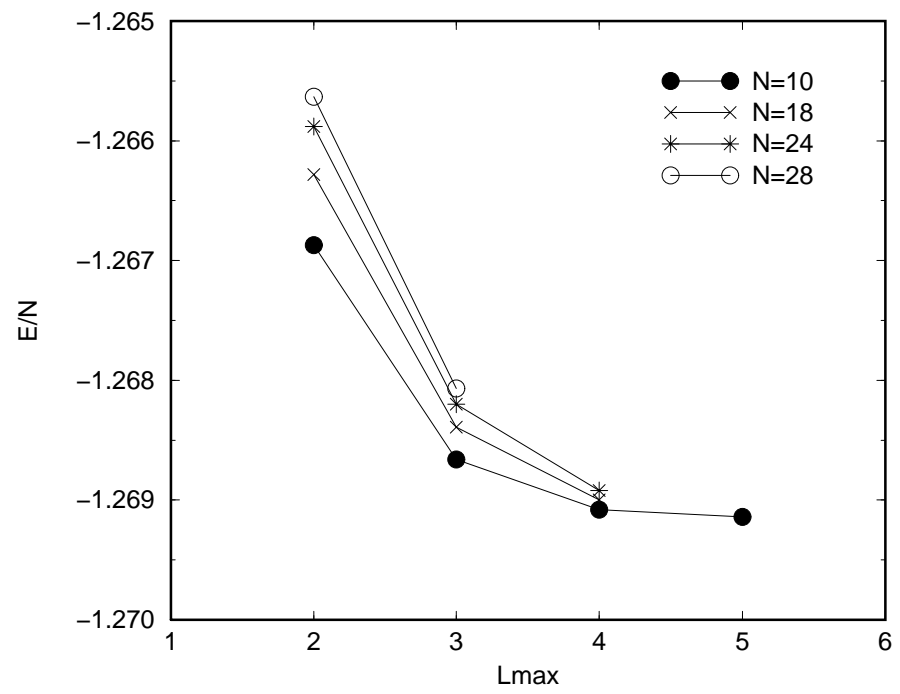

Figure 3: Ground state energy per site for the standard Hubbard model on ladders versus the maximum value of $L$, which is denoted by $L_{\max }$, calculated for several values of the system size $N$. Lines in the figure are to guide eyes. Values of the parameters in the Hamiltonian are $t_{c}=1$, $t_{r}=2$ and $U=4$. 


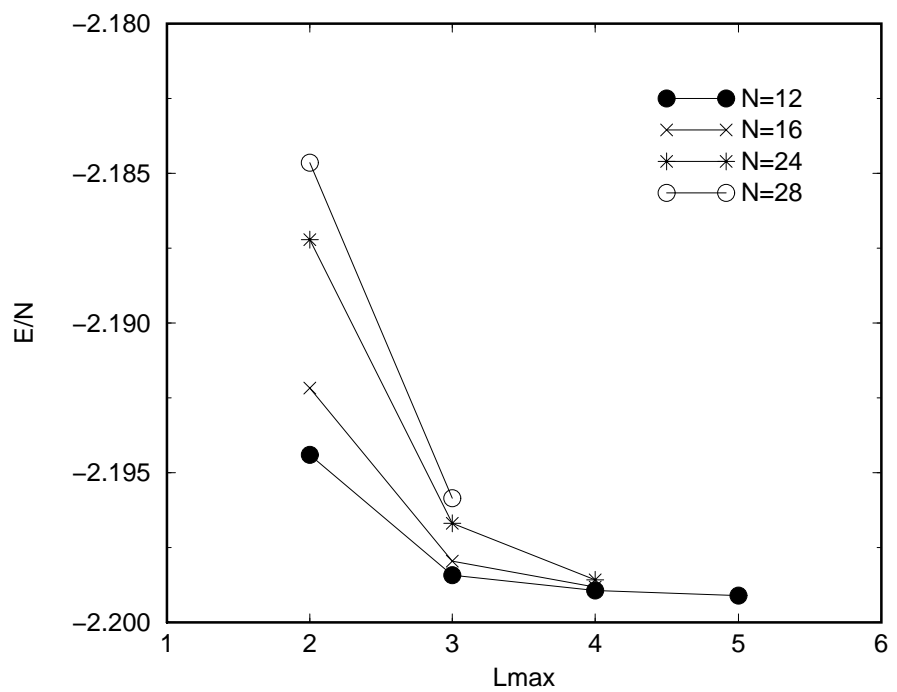

Figure 4: Ground state energy per site for the extended Hubbard model described by (12) on ladders with size $N$ varying up to 28 , as a function of $L_{\max }$. Lines in the figure are to guide eyes. Values of the parameters in the Hamiltonian are $t_{c}=1, t_{r}=2, V_{c}=2, V_{r}=-4$ and $U=4$. 